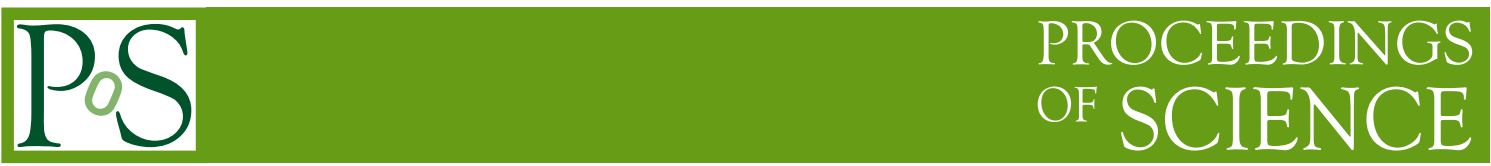

\title{
R\&D towards future upgrade of the CMS RPC system
}

\section{G. Pugliese*}

On behalf of the CMS Muon Group

Author affiliation: Dipartimento Interateneo di Fisica and Sezione INFN di Bari, Via G.

Amendola 173, 70126 Bari, Italy

E-mail: gabriella.piugliesedcern.ch

The CMS experiment at the CERN Large Hadron Collider (LHC) is equipped with a redundant muon trigger system based on Drift Tubes Chambers (DT) in the barrel region and Cathode Strip Chamber (CSC) in the endcap regions and Resistive Plate Chambers (RPC) in both regions up to $|\eta|=1.6$. In view of the High Luminosity LHC (HL-LHC) phase, to keep the same muon performance new muon stations will be installed in the forward region $(1.6<|\eta|<2.4)$ and in the very forward region up to $|\eta|=2.8$. The 3 th and 4 th stations will be equipped with a new generation of RPC with improved performance (iRPC), capable of handling the challenging conditions expected at the HL-LHC. An extensive R\&D program has been undertaken to define the detector design and related electronics that meet the HL-LHC requirements. A summary of iRPC performance results is here reported.

38th International Conference on High Energy Physics 3-10 August 2016

Chicago, USA

${ }^{*}$ Speaker. 


\section{Introduction}

The CMS Muon detector system consists of three types of detectors: Drift Tubes (DT) in the barrel region, Cathode Strip Chambers (CSC) in the endcap and Resistive Plate Chambers (RPC) in both regions up to $|\eta|=1.6$. The system was designed to provide muon identification, excellent triggering, timing and momentum measurements at the LHC up to a luminosity of $1 \cdot 10^{34} \mathrm{~cm}^{-2} \mathrm{~s}^{-1}$. The CMS RPC system is one of the largest ever built detector employing the RPC technology with a total coverage area of about $5000 \mathrm{~m}^{2}$. The CMS chamber has a double-gap design, consisting of two 2-mm-thick resistive $\left(2 \div 5 \cdot 10^{10} \Omega \mathrm{cm}\right)$ High Pressurised Laminate (HPL) plates separated by a 2-mm-thick gas gaps. Readout strips are in between the two gas gaps. The RPCs are operated in avalanche mode with a 3-component, non-flammable, gas mixture that consists of $95.2 \%$ of $\mathrm{C}_{2} \mathrm{H}_{2}$ $\mathrm{F}_{4}, 4.5 \%$ of $\mathrm{i}-\mathrm{C}_{4} \mathrm{H}_{10}$, and $0.3 \%$ of $\mathrm{SF}_{6}$ with a relative humidity of $40 \% \div 50 \%$ [1]. During the first five years of LHC data taking the performance of the muon systems has proved to be outstanding [2]. In the second phase of the LHC physics program, the accelerator will increase the luminosity up to $5 \cdot 10^{34} \mathrm{~cm}^{-2} \mathrm{~s}^{-1}$, providing to CMS an additional integrated luminosity of about $3000 \mathrm{fb}^{-1}$ over 10 years of operation, starting in 2025. To keep the same performance at HL- LHC conditions new muon stations will be installed in the forward region $(1.6<|\eta|<2.4)$ and in the very forward region up to $|\eta|=2.8$. The 3th and 4th stations will be equipped with a new generation of RPC with improved performance, capable of handling the challenging conditions expected.

\section{New detector requirements at HL - LHC}

To fulfill the HL-LHC requirements, the new chambers have to be certified for a stable performance up to an integrated luminosity of $3000 \mathrm{fb}^{-1}$, at a maximum rate about $2 \mathrm{kHz} / \mathrm{cm}^{2}$, including a safety factor of 3 . A spatial resolution of $\sim 2-3 \mathrm{~mm}$ is needed to improve muon identification and momentum resolution, i.e. by a factor 4-6 compared to the present system. An high timing resolution $\sim 100$ ps would be favourable for background and pileup rejection and to provide a discriminating signature for hypothetical heavy stable charged particles and allow for corresponding mass determination [3]. Since 2014, the RPC group is involved in an extensive R\&D program to define the detector design and electronics that meet these requirements. To overcome the limited particle rate capabilities of the present RPC, all relevant detector improvement factors are being investigated. A new electrode material, the low resistive silicate glasses produced in China [4], has been also considered and a lower resistivity range $\left(\sim 10^{10} \mathrm{~cm}\right)$ has been used since the rate capability is inversely proportional to the resistivity of the electrodes. The gas gap thickness has been reduced in order to reduce the average avalanche charge and a new generation Front-End Board electronics used in order to move part of the amplification to the electronics. A lower average charge is crucial to enhance the higher rate capability and certainly reduce the aging guaranteeing the longevity of the detector. New detector geometry has been considered with reduced electrode thickness $(2 \div 1 \mathrm{~mm})$ and number (multi-gap-like geometry).

\section{Rate capability}

A double gap trapezoidal prototype RPCs $\left(\sim 1 \mathrm{~m}^{2}\right)$, with a gas gap thicknesses of $1.6 \mathrm{~mm}$, was built and tested at Korea University. In 2015, the prototype was tested at the CERN Gamma 
Irradiation Facility (GIF++) where an high energy muon beam $(150 \mathrm{GeV})$ combined with a 14 $\mathrm{TBq}{ }^{137} \mathrm{Cs}$ gamma source and a set of moveable filters allows to study the detector performance as a function of the gamma background. The efficiency for double-gap RPCs as a function of the applied HV for different gamma background conditions is shown in Fig. 1a. No significant degradation of chamber performance was observed up to $1 \div 1.5 \mathrm{kHz} / \mathrm{cm}^{2}$. More results can be found here [5].

An RPC telescope with five single gaps $\left(30 \times 30 \mathrm{~cm}^{2}\right)$, four with Chinese low resistive glass and one with float glass, was built at IPNL (Lyon) and tested at the CERN SPS H4 line muon beam and at GIF++ in 2015. The signals were collected using a PCB electronics plate equipped with $1 \times 1 \mathrm{~cm}^{2}$ readout pads and readout by a 64-channel HARDROC ASICs [6]. In Fig. 1b, gap efficiency as a function of the gamma flux is showed in blue and in red for the low and float glasses, respectively. A 70\% single-gap efficiency for low resistivity GRPC at a flux of $2 \mathrm{kHz} / \mathrm{cm}^{2}$ (i.e. > $90 \%$ double-gap efficiency) was measured.
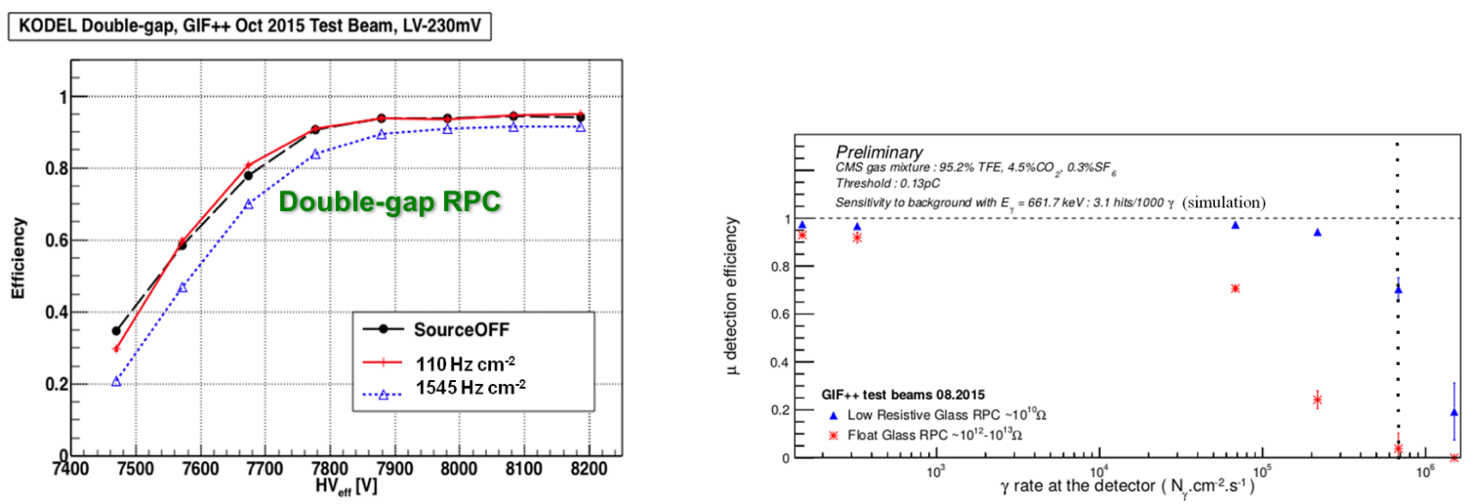

Figure 1: Left: Efficiency for double-gap RPCs as a function of the applied HV for different gamma background conditions. Right: Glass gap RPC efficiency as a function of the gamma flux.

\section{Spatial and time resolution}

A prototype $30 \times 30 \mathrm{~cm}^{2}$ with a gas gap of $1.2 \mathrm{~mm}$ separated by two $1 \mathrm{~mm}$ Chinese glasses was built at IPNL (Lyon) and tested at the CERN PS in 2014. The signal was collected by two layers of 128 strips with a pitch is $2.5 \mathrm{~mm}$ and readout by the HARDROC ASICs. The two layer strips are shifted by $1 \mathrm{~mm}$ each other in the direction perpendicular to the strips one. This configuration is designed to increase the spatial resolution by looking at the coincidence of fired strips. Spatial resolution as a function of the rate was measured by looking at the coincidences of strips in both layers is showed in Fig. 2 a. The resolution improves from 1.5 down to $1 \mathrm{~mm}$ at $10 \mathrm{kHz} / \mathrm{cm}^{2}$, according to the reduction of strip multiplicity.

The dimension of the Chinese low resistive glasses is limited to $33 \times 28 \mathrm{~cm}^{2}$ by processing technology. To obtain a CMS size RPC, a mosaic design has been adopted with glass pieces joined tougher with a fishing line block [4]. A multi gap prototype (MRPC) with five gas gaps of $250 \mu \mathrm{m}$ width, with a dimension of $25 \times 67 \mathrm{~cm}^{2}$, was tested at the electron accelerator ELBE at HZDT in Germany. The flux of the primary beam is tuneable from few to $10^{7}$ electrons/s. Efficiency, time 

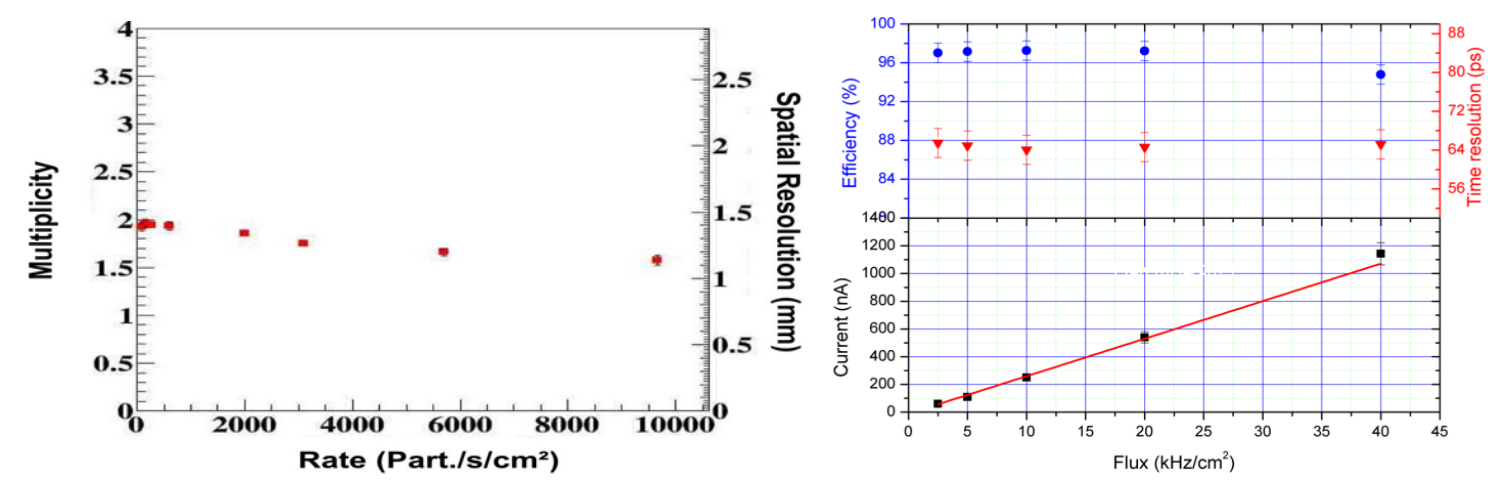

Figure 2: Left: Strip multiplicity and spatial resolution as a function of the beam flux. Right: Efficiency, time resolution and current as function of the beam flux.

resolution and current as function of the beam flux are showed in Fig. 2 b. Efficiency of about 95 $\%$ and a time resolution of $\sim 60 \mathrm{ps}$ were measured almost constant up to a flux rate of $40 \mathrm{kHz} / \mathrm{cm}^{2}$.

\section{Conclusions}

A new generation of RPC using low resistivity electrodes (glass and HLP) has been extensively tested during the past years by the CMS RPC Group. It has been demonstrated that the iRPC can sustain the background foreseen rates at HL-LHC. Furthermore, the use of new detector design and electronics has allowed to achieve high spatial resolution $(\sim 1 \div 1.5 \mathrm{~mm})$ and excellent time resolution ( $\sim 60 \mathrm{ps})$. Further tests on full-size chambers equipped with final electronics will begin this year at GIF++ to study detector longevity for the whole HL-LHC operation period.

\section{Acknowledges}

We would like to thank CERN EN and PH divisions for the support during test beams and GIF++ operation. This project is supported by the Italian "Ministero degli Affari Esteri e della Cooperazione Internazionale" and by the H2020 project AIDA-2020, GA no. 654168.

\section{References}

[1] CMS Coll. "The Muon Project TDR", CERN/LHCC 97-32, Dec 1997

[2] CMS Coll. "The performance of the CMS muon detector using pp collisions at $\sqrt{s}=7 \mathrm{TeV}$ at the LHC", MUO-11-001

[3] CMS Coll. "Phase 2 Technical proposal" CERN/LHCC-2015-010

[4] F. Wang et al. "Performance study of a Mosaic high rate MRPC", JINST 11 (2016), C09016

[5] K.S. Lee et al. "Radiation tests of real-size prototypes RPCs for the phase2 Upgrade of the CMS Muon system", JINST 11 (2016), C08008

[6] F. Dulucq et al., HARDROC: "Readout chip for CALICE/EUDET Digital Hadronic Calorimeter", 2010 IEEE, pp 1678-1683 2010 